\title{
Tratamiento quirúrgico de duplicidad peneana completa
}

\author{
Carvalho AP, Ramires R, Soares J, Carvalho La Fuente, Filinto M. \\ Servicio de Urologia. Hospital General de Santo António. Oporto, Portugal.
}

Actas Urol Esp. 2008;32(9):941-944

\section{RESUMEN}

TRATAMIENTO QUIRÚRGICO DE DUPLICIDAD PENEANA COMPLETA

La duplicidad peneana es una anomalía rara, con una incidencia de 1 entre 5.500.000. Normalmente está asociada a otras malformaciones como duplicidad vesical, cloaca, ano imperforado, duplicidad de recto y sigma y a deformidades vertebrales.

Los autores presentan la técnica quirúrgica que aplicaron para la resolución de un caso raro de duplicidad peneana completa en un individuo de sexo masculino, de cuatro años de edad, sin otras malformaciones sistémicas asociadas.

Palabras clave: Duplicidad peneana. Cirugía reconstructiva.

\section{ABSTRACT \\ SURGICAL TREATMENT OF COMPLETE PENILE DUPLICATION}

Penile duplication is a rare anomaly with an incidence of 1 in 5,500,000. It is almost associated with other malformations like double bladder, presence of the cloaca, imperforate anus, duplication of the recto sigmoid and vertebral deformities.

The authors present the surgical technique to resolve a rare case of complete penile duplication in a 4 years old child, without any other malformation.

Keywords: Penile duplication. Reconstructive surgical procedures.

$\mathrm{L}$ duplicidad peneana completa es una anomalía congénita rara, existiendo tan sólo 100 casos descritos en la literatura. Esta malformación puede afectar a la totalidad o a parte del pene. La orina se puede eliminar por uno o por los dos penes. Embriológicamente parece asociarse a una alteración en las capas mesodérmicas ${ }^{1}$.

\section{CASO CLÍNICO}

Presentamos el caso clínico de un niño de 4 años, visitado en la consulta de Urología desde su nacimiento por presentar duplicidad peneana completa.

El niño era de raza caucasiana, hijo de una pareja joven, sin consanguinidad y sin historia de patología hereditaria ni de otras patologías conocidas. Embarazo vigilado, no se detectaron problemas. SerologÍa de sífilis, hepatitis y VIH negativas. La madre era inmune a la rubéola pero no lo era a la toxoplasmosis. Calendario vacunal actualizado. Parto distócico a las 39 semanas de gestación, sin complicaciones durante el parto o en el post parto. Apgar 9/10. Peso al nacimiento de $3780 \mathrm{gr}$ (p75), altura 51,5cm (p75) y perímetro cefálico de $35,5 \mathrm{~cm}$ (p50). En el examen clínico que se efectuó al nacer se detectó la presencia de dos penes (Fig. 1), el izquierdo de tamaño normal, el derecho más rudimentario. Los dos glandes estaban cubiertos por el mismo pliegue cutáneo (Fig. 2), no se detectaron otras anomalías asociadas. El ecocardiograma y la ecografía transfontanelar no mostraron ninguna alteración. El estudio hormonal y el cariotipo eran normales.

Cistouretrografía retrógrada permiccional (Fig. 3) mega uretra con estenosis uretral desde ese punto, asociada al pene mas rudimentario, el derecho. Comunicación de las dos uretras con la vejiga. 


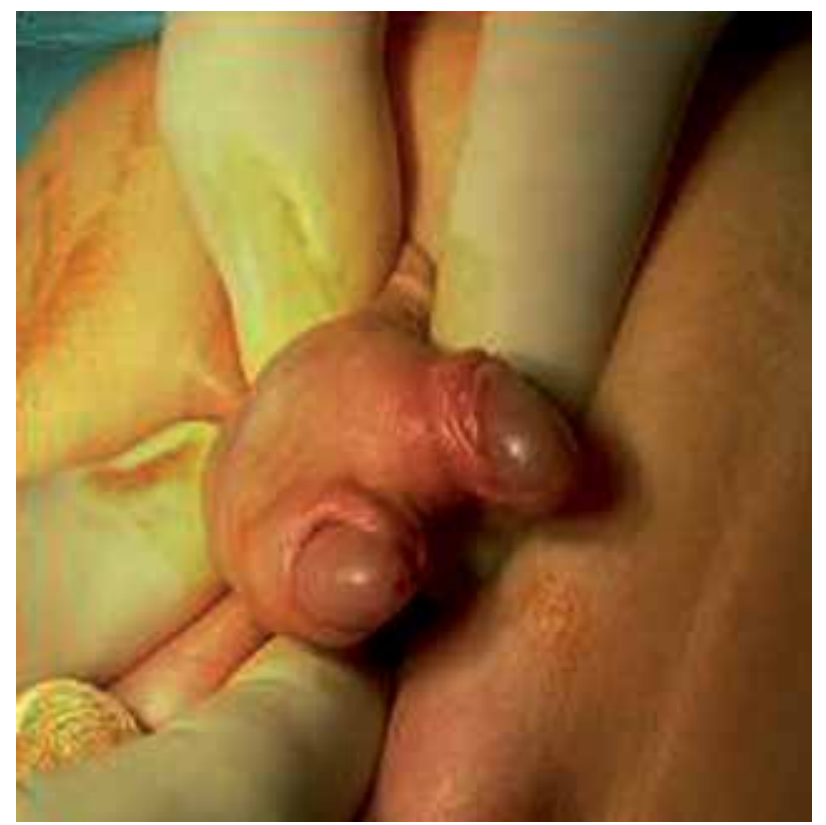

FIGURA 1

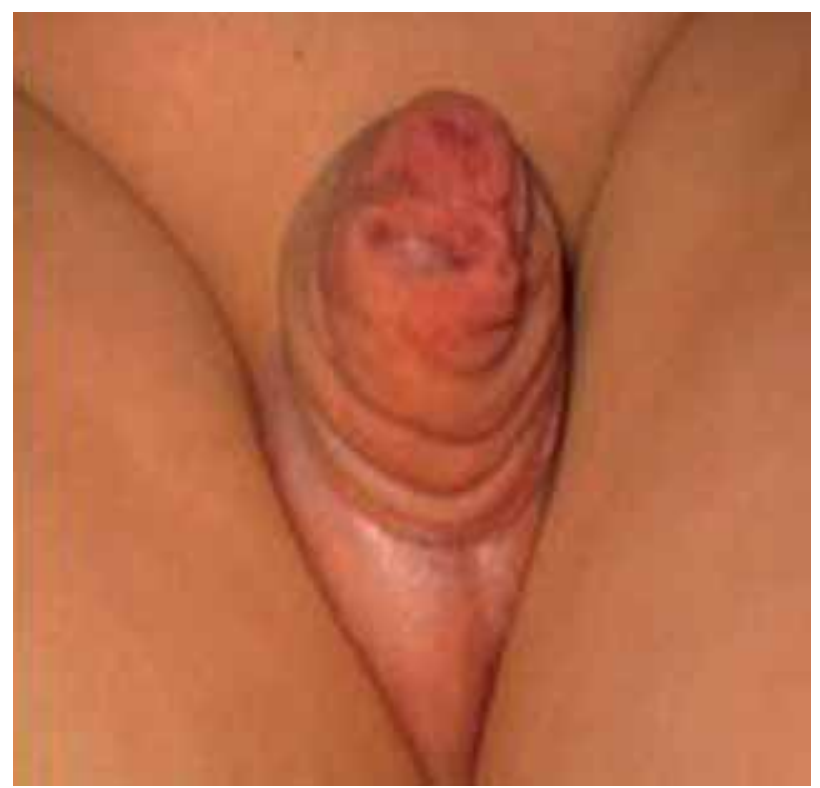

FIGURA 2

Ecografía peneana- se observa dos cuerpos cavernosos, un cuerpo esponjoso y una uretra en cada uno de los penes.

No hubo episodios de infección. Capacidad eréctil preservada en los dos penes. La uretra sólo era permeable en el pene izquierdo, la derecha se cerró casi por completo de manera espontánea.

La resonancia magnética realizada a los cuatro años constató la presencia de 2 cuerpos cavernosos y de uno esponjoso de aspecto normal en el pene

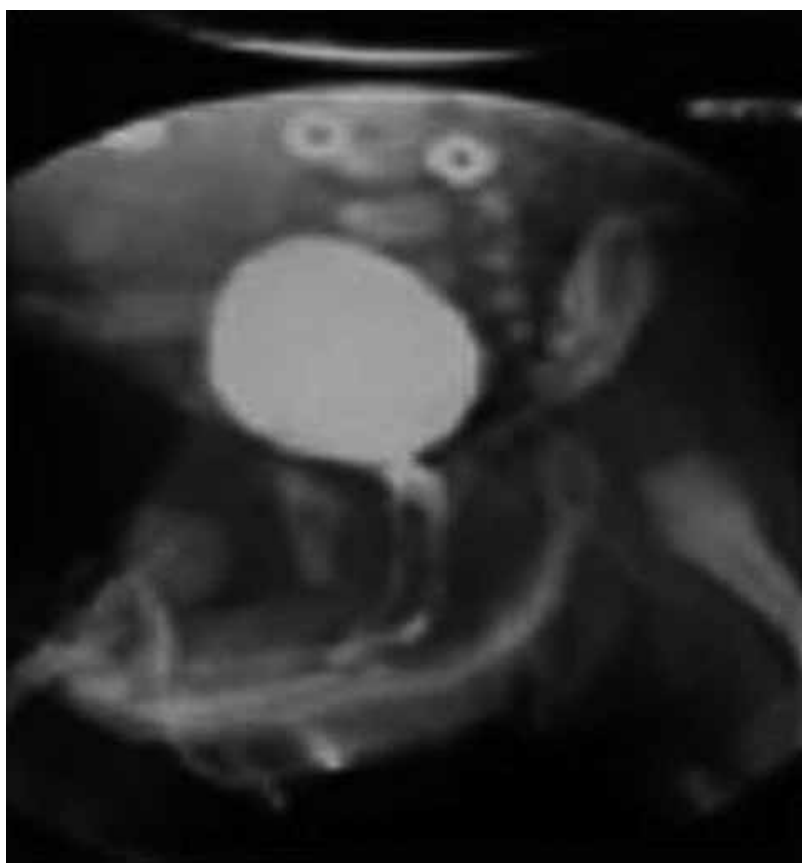

\section{FIGURA 3}

izquierdo y de un cuerpo cavernoso normal y otro cavernoso junto con uno esponjoso atróficos en el pene derecho (Fig. 4)

Se optó por realizar la disociación de los penes hasta donde se insertan los cuerpos cavernosos (Fig. 5), verificando la permeabilidad de las dos uretras. Se colocó una cistostomía. Se amputó a nivel de la raíz del pene derecho y se anastomosó la ure-

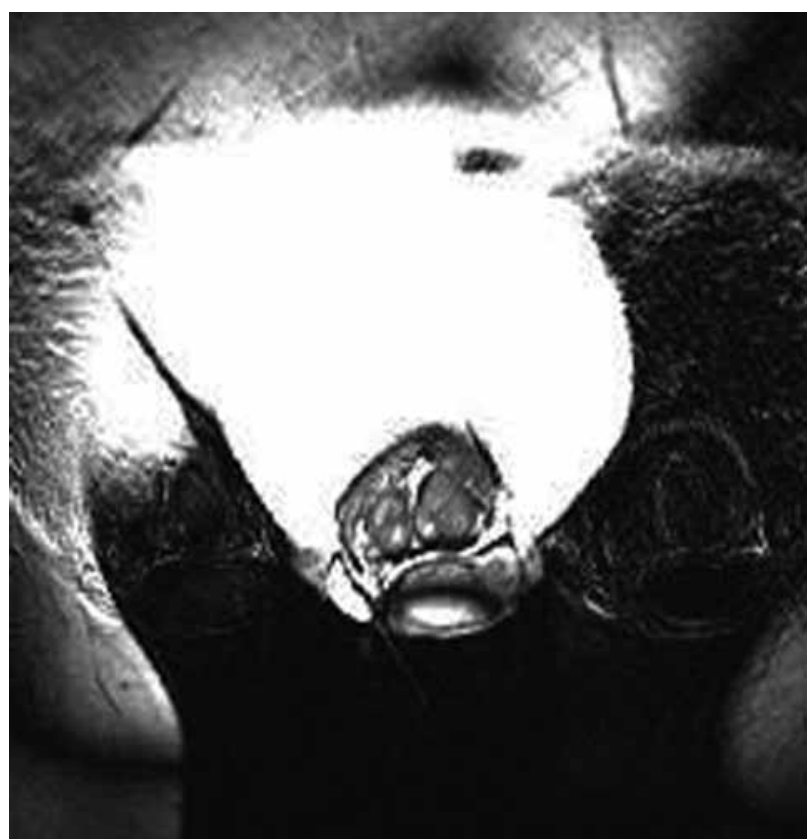

FIGURA 4 


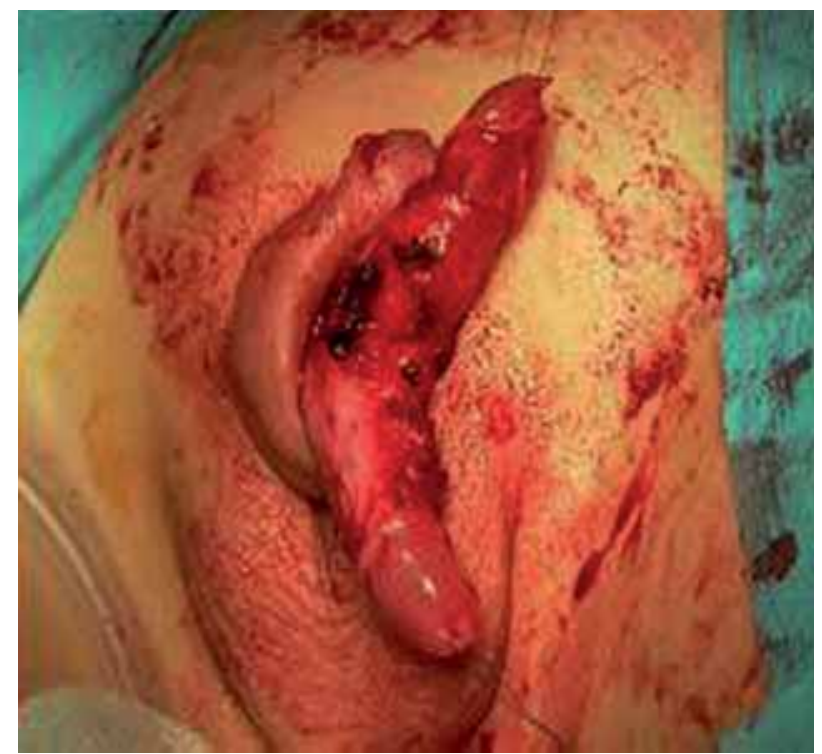

FIGURA 5

tra derecha, de forma termino-lateral, a la uretra izquierda (Fig. 6). Se realizó una plastia de la piel sobrante (Fig. 7). Tres meses después de la intervención, el paciente se encuentra bien y tiene micciones espontáneas.

\section{DISCUSIÓN}

La duplicidad peneana tiene diversas variantes que van desde un pequeño pene accesorio hasta la duplicidad peneana completa.

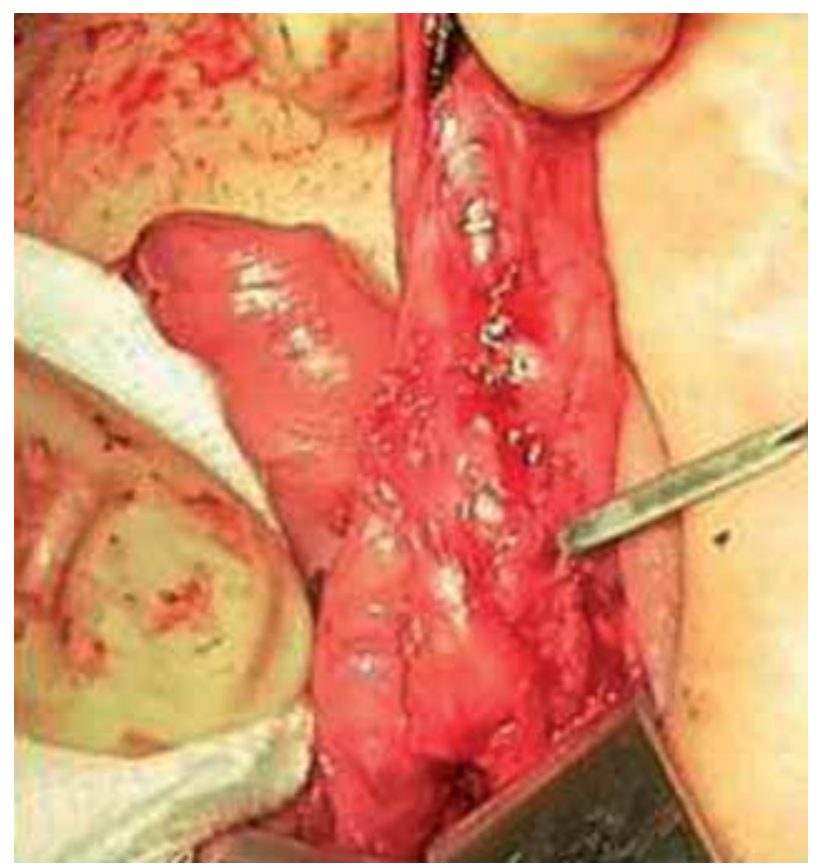

FIGURA 6

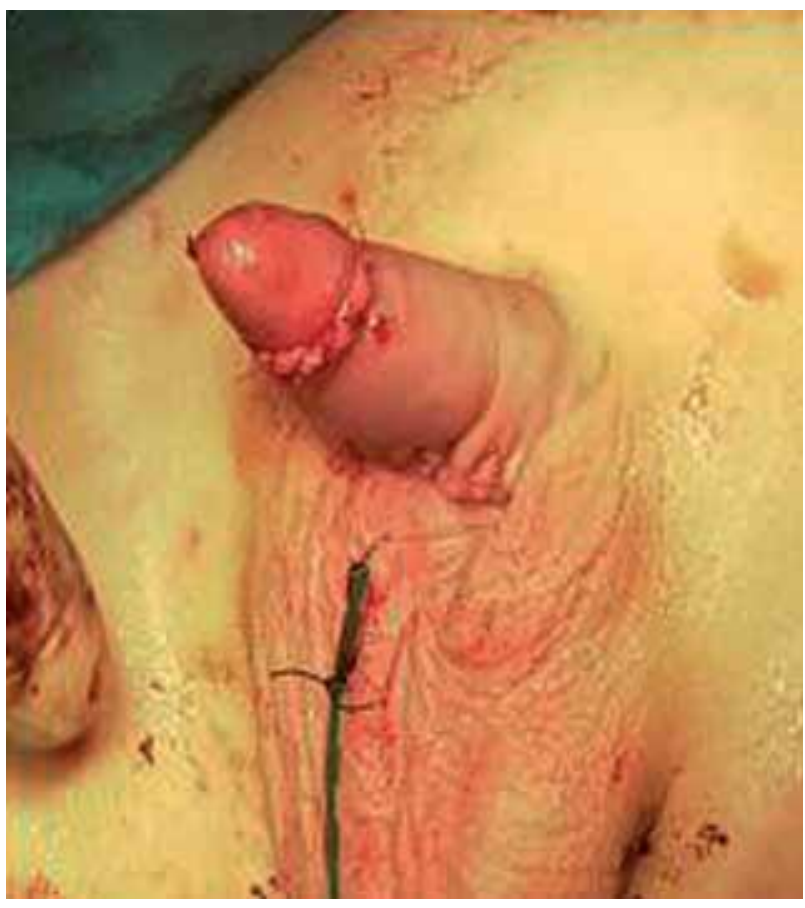

FIGURA 7

La difalia verdadera es la variante menos frecuente de la duplicidad peneana, caracterizándose, normalmente, por la presencia en paralelo de los dos penes (duplicidad transversal) o uno encima del otro (duplicidad longitudinal). Normalmente cada pene tiene un cuerpo esponjoso y dos cavernosos ${ }^{2}$.

Normalmente estas anormalidades se asocian a otras más extensas, como por ejemplo, dentro de las más frecuentes, duplicidad vesical, extrofia vesical, ano imperforado, agenesia o atrofia renal, escroto bífido y duplicación cólica entre otras ${ }^{3}$.

El desarrollo embriológico de esta malformación es escaso ya que hay varias causas para los diversos tipos de duplicidad y además como hay pocos casos descritos no es fácil atribuir causas comunes a todos ellos.

Gyftopoulos et al. analizaron 77 casos publicados en la literatura de duplicidad peneana con referencias a aspectos embriológicos y llegaron a la conclusión que no había semejanzas entre ellos ${ }^{4}$. A pesar de ello, hay autores que defienden que hay un tipo de desajuste en la terminación de la membrana cloacal y una relación con el tubérculo genital y con el seno urogenital ${ }^{5}$.

La función eréctil en los casos de duplicidad peneana varía significativamente. Normalmente uno o incluso los dos penes tienen capacidad eréctil. En 
casos de duplicidad peneana completa en edad adulta se describieron erecciones conjuntas y ocasionalmente eyaculación ${ }^{3}$.

La cistouretrografía retrógrada permiccional debe ser una prueba diagnostica realizada precozmente para poder constatar la permeabilidad de ambas uretras y su continuidad con la vejiga ya que es frecuente esta alteración asociarse a malformaciones vesicales, a parte de para permitir una mejor planificación en su posterior corrección quirúrgica, con el fin de evitar posibles complicaciones posteriores, como por ejemplo las fístulas.

La RMN nos permite tener una excelente apreciación anatómica de las complejas anomalías genitales, ayudando al diagnostico y maximizando los resultados de un tratamiento quirúrgico. Hay autores que defienden que este debe ser el procedimiento diagnostico de elección para evaluar las malformaciones congénitas de los órganos genitales.

Los objetivos del tratamiento quirúrgico son: separación de los tractos urogenitales, preservación de la continencia y de la función eréctil y reconstrucción de los genitales externos de una manera funcional y estética.

\section{CONCLUSIÓN}

El tratamiento debe ser orientado de manera individualizada, las malformaciones asociadas se deben tratar en primer lugar, sobretodo si compro- meten la vida del paciente, por lo que se hace prioritario llegar a un diagnóstico correcto de las anomalías y de la anatomía de cada pene.

\section{REFERENCIAS}

1. Berrocal T, López-Pereira P, Arjonilla A, Gutiérrez J. Anomalies of the distal ureter, bladder and urethra in children: embriologic, radiologic and pathological features. Radiographics. 2002;22(5):1139-1164.

2. Djordjevic ML, Perovic SV. Complex penile joining in a case of wide penile duplication. The Journal of Urology. 2005;173(2): 587-588.

3. Erdil H, Mavi A, Erdil S, Gumusburun E. Urethral Duplication case report. Acta Med Okayama. 2003;57(2):91-93.

4. Gyftopoulos K, Wolffenbuttel KP, Nijman RJ. Clinical and embryologic aspects of penile duplication and associated anomalies. Urology. 2002;60(4):675-679.

5. Djordjevic ML, Perovic SV. Complex penile joining in a case of wide penile duplication. The Journal of Urology. 2005;173(2): 587-588.

6. Lapointe SP, Wei DC, Hricak H, Varghese SL, Kogan BA, Baskin LS. Magnetic resonance imaging in the evaluation of congenital anomalies of the external genitalia. Urology. 2001;58(3):452456.

Correspondencia autor: Dr. AP Fernandes Carvalho Serviço de Urologia

Hospital de S. Marcos, Apartado 2242,

4700 Braga (Minho) Portugal.

Tel.: +351253209110

E-mail autor: antoniopedrocarvalho@gmail.com

Información artículo: Nota clínica

Trabajo recibido: marzo 2007

Trabajo aceptado: julio 2007 